\title{
An Appraisal of UNIVAUTO - The First Discovery Program to Generate a Scientific Article
}

\author{
Vladimir Pericliev \\ Institute of Mathematics and Informatics, bl.8, 1113 Sofia, Bulgaria \\ peri@math.bas.bg
}

\begin{abstract}
In a companion paper ([14]), I describe UNIVAUTO (UNIVersals AUthoring TOol), a linguistic discovery program that uncovers language universals and can write a report in English on its discoveries. In this contribution, the system is evaluated along a number of parameters that have been suggested in the literature as necessary ingredients of a successful discovery program. These parameters include the novelty, interestingness, plausibility and intelligibility of results, as well as the system's portability and insightfulness.
\end{abstract}

\section{Introduction}

In a companion paper ([14]), I describe UNIVAUTO (UNIVersals AUthoring TOol), a system whose domain of application is linguistics, and in particular, the study of language universals, an important trend in contemporary linguistics. Given as input information about languages presented in terms of feature-values, (eventually) the discoveries of another human agent arising from the same data, as well as some additional data, the program discovers the universals in the data, compares them with the discoveries of the human agent and, if appropriate, generates a report in English on its discoveries.

Running UNIVAUTO, with different queries, on the data from a classical paper by Greenberg [4] on word order universals, the system has produced several linguistically valuable texts, one of which was submitted for publication to a refereed linguistic journal without any further human editing (except for the formatting needed to conform to the style-sheet of the journal), and without disclosing the "machine origin" of the article. The article was accepted for publication ([11]). Another of these texts was also published with no post-editing as [13]. Exploring the phonological database UPSID ([8],[9]), the system has generated about 30 pages of text, comprising phonological universals and their support, which is included - as outputted by the system - in [16] (a part of these universals has already been published at the Universals Archive at the University of Konstanz (http://ling.uni-konstanz.de/pages/proj/sprachbau.htm). ${ }^{1}$

${ }^{1}$ Some further discoveries, in which the human user played an appreciable part, will be mentioned in Sect. 3.6. 
In this contribution, the system is evaluated along a number of parameters that have been suggested in the literature as necessary ingredients of a successful discovery program. These parameters are the novelty, interestingness, plausibility and intelligibility of results, as well as the system's portability and insightfulness. The overview of the system in the next section provides the necessary context for the following discussion.

\section{Overview of UNIVAUTO}

UNIVAUTO operates in the domain of language universals, a branch of linguistic typology studying the common properties (=universals) shared by all or most of the languages of the world. ${ }^{2}$

Some familiar examples are "All languages have oral vowels" (unconditional, non-statistical universal), "If a language has a dual number, it has a plural" (implicational (or conditional), non-statistical universal), "In most languages, if they have the order Verb-Subject-Object, then the adjective follows the noun" (implicational, statistical universal).

UNIVAUTO accepts as INPUT the following, manually prepared, information:

1. A database (=a table), usually comprising a sizable number of languages, described in terms of some properties (feature-value pairs), as well as a list of the abbreviations used in the database. The program also knows their "names", or what the abbreviations used for feature values stand for. A special value can occur in a database, designating either that the corresponding feature is inapplicable for a language or that the value for that feature is unknown.

2. A human agent's discoveries, arising from the same database, stated in terms of the used abbreviations.

3. Other information. Aside from these two basic sources of information, the input includes also information on: the origin of database (the full citation of work where the database is given); reference name(s) of database; language families and geographical areas to which the languages in the database belong; etc.

The system supports various QUERIES. Thus, the user may require different: (i) logical types of universals (unconditional or implicational with two or more variables), (ii) minimum number of supporting languages, (iii) percentage of validity and (iv) statistical significance. The user can also choose the minimum number of (v) language families and (vi) geographical areas the supporting languages should belong to.

\footnotetext{
${ }^{2}$ For a recent introduction, cf. e.g. [2]. Also, cf. several journals, incl. the authoritative Linguistic Typology, proclaiming as one of its goals the publication of universals register, as well as the electronic Konstanz Universals Archive, containing around 2000 universals from all linguistic levels that have been collected from the published literature.
} 
UNIVAUTO is a large program, comprising two basic modules: one in charge of the discoveries of the program, called UNIV(ersals), and the other in charge of the verbalization of these discoveries, called $\mathrm{AU}$ (thoring) $\mathrm{TO}(\mathrm{ol})$.

UNIV can discover various non-redundant logical patterns (universals), supported in user-specified thresholds of languages, language families and geographical areas, percentage of validity and statistical significance. Importantly, given the discoveries of another, human agent, UNIV employs a diagnostic program to find (eventual) errors in the humanly proposed universals. Currently, the system identifies as PROBLEMS the following categories:

- Restriction Problem: Universals found by human analyst that are below a user-selected threshold of positive evidence and/or percentage of validity and/or statistical significance.

- Uncertainty Problem: Universals found by human analyst that tacitly assume a value for some linguistic property which is actually unknown or inapplicable.

- Falsity Problem: Universals found by human analyst that are false or logically implied by simpler universals.

The DISCOVERIES of UNIV fall into two types: (1) a list of new universals, and (2) a list of problems (sub-categorized as above).

UNIV assesses the "scientific merit" of its discoveries in order to decide whether to generate a report or not. It uses a natural and simple numeric method: UNIV's discoveries (novel universals plus problems) are judged worthy of generating a report if they are at least as many in number as the number of the published discoveries of the human agent studying the same database.

The authoring module AUTO follows a FIXED SCENARIO for its discourse composition, whose basic components are: (1) Statement of title, (2) Introduction of goal, (3) Elaboration of goal, (4) Description of the investigated data and the human discoveries, (5) Explaining the problems in the human discoveries, (6) Statement of the machine discoveries, (7) Conclusion. The details of this scenario, however, will vary in accordance with a number of parameters, related to the specific query to the system and the corresponding discoveries made.

We cannot go into details here, and will only mention that for its SURFACE GENERATION, AUTO employs a hybrid approach, using both templates and rules, which are randomly chosen among a set of alternatives in order to ensure intra-textual variability (for details, cf. [14]).

\section{Evaluating UNIVAUTO}

A common pragmatic criterion for evaluating discovery systems is the publication of their discoveries in the specialized domain literature. According to this criterion, UNIVAUTO performs well: its outputs have found outlet in several linguistic publications ([11], [13], [15], [16] (the latter under submission)).

Valdés-Pérez [20] has alternatively characterized machine scientific discovery as the generation of novel, interesting, plausible, and intelligible knowledge, and 
has suggested that a successful system should ideally have all these capacities. We have also mentioned as advantageous to discovery systems the features of portability and insightfulness, which were found to be common to four linguistic discovery systems that the author has been involved in ([12]). Below I describe UNIVAUTO along these six dimensions. (Cf. also [1] for an interesting similar discussion concerning basically systems in the domain of mathematics).

\section{$3.1 \quad$ Novelty}

UNIVAUTO has so far produced around 60 pages of text, covering about 250 new universals from the fields of word order and phonology. It has found (cf. [11], [13]) that two of the proposed word order universals in the classical article by Greenberg [4] are actually false and that seven others are exceptionless relative to the database investigated rather than statistical, as claimed by Greenberg. Three other of Greenberg's ordering universals were shown to tacitly assume feature values for some languages which are actually unknown to the database. All these circumstances have remained unnoticed by previous human researchers, and ironically, some of the problematic universals are widely disseminated in the linguistic community (cf. e.g. the complete enumeration of Greenberg's [4] ordering universals in The Linguistics Encyclopedia, London and N.Y., 1991).

Inspecting two further word order databases from Greenberg [4] and Hawkins [6], which are really small $24 \times 4$ tables, the system also managed to find patterns that have escaped these authors, considered to be the authorities in the field in the textbook by Croft [2, page 57]. (Cf. also Sect. 3.6.)

Similarly, many novel phonological universals were found in the UPSID database in comparison with Maddieson's [8] findings, as well as some problems in these and other related proposals in the literature (lack of statistical significance and/or low level of validity and/or insufficiently diverse language support). Cf. $[16]$.

Three design properties of the system enhance the chances of finding novel knowledge. The first is the system's ability to explicitly check its own discoveries against those of a human agent exploring the same data. More generally, this strategy is not impractical in a linguistic discovery system on universals in view of the availability of universals archives, such as the Konstanz Archive mentioned above. The second is the exhaustive search of a combinatorial space that the system performs. Such comprehensive searches of combinatorial spaces, that are furthermore dense with solutions, are known to be very difficult, if not completely beyond the reach of a human investigator, a trite circumstance in computer science (but, unfortunately, not so in many domain sciences as linguistics). As a corollary of the exhaustive search, the system can make meta-scientific claims to the effect that "These are all universals of the studied type (relative to the database)". The third design property is the ability of the system to handle diverse queries (esp. those concerning different logical types of universals), some of which may not have been seriously posed or pursued before. 


\subsection{Interestingness}

The interestingness of UNIVAUTO's findings is partly derived from the interestingness of the task it automates. Indeed, linguistics has always considered the discovery or falsification of a universal an achievement.

From a purely design perspective, the system attempts to enhance the discovery of interesting universals by outputting only the stronger claims and discarding the weaker ones. Thus, if Universal 1 logically implies Universal 2, the first is retained and the second is ignored. E.g. "All languages have stops" implies "If a language has a fricative it also has a stop" and the second claim must therefore be dismissed as a pseudo-universal. (Ironically, this claim has been actually made more than 60 years ago in a celebrated book by Jakobson [7], another linguistic luminary, and has never been refuted.)

\subsection{Plausibility}

The plausibility of posited universals has been a major concern for UNIVAUTO. Universals are inductive generalizations from an observed sample to all human languages and as such they need substantial corroboration. The system disposes with two principled mechanisms to this end. The first is the mechanism ensuring statistical plausibility, allowing the user to specify a significance threshold for the system's inferences. It is embodied in two diverse methods, the chi-square test and the permutation test ${ }^{3}$, which can alternatively be used. The second plausibility mechanism pertains to the need for qualitatively different languages to provide support for a hypothetical universal for it to be outputted by the program. The specific measure of "typological diversity" of the supporting languages is chosen by the user of the system, by selecting the minimum number of language families and geographical areas to which the supporting languages must belong.

The plausibility of (eventual) criticisms of a human agent's discoveries is even less problematic. Indeed, one can definitely (and not only plausibly) say when a proposition is false relative to a known database, and that is exactly what the system does.

\subsection{Intelligibility}

With some discovery systems, the user/designer may encounter difficulties in interpreting the program's findings. With other systems, typically those that model previously defined domain-specific problems, and hence systems searching conventional problem spaces, the findings would as a rule be more intelligible. However, intelligibility is a matter of degree and UNIVAUTO seems unique in producing an understandable English text to describe its discoveries (but see also [5]).

The following excerpt from [11] will suffice to give an idea of the system's output:

\footnotetext{
${ }^{3}$ UNIVAUTO permutation test is that presented in [18].
} 
We confirmed the validity of $<$ Greenberg's $>$ universals [12,13,15-a,15-b,21-a,22$a, 27-a]$.

$<$ Greenberg's $>$ Universals [16-a,16-b,16-c] are uncertain, rather than indisputably valid in the database investigated, since they assume properties in languages, which are actually marked in the database as "unknown or inapplicable". ... Universal [16-a] would hold only if the feature AuxV/VAux is applicable for Berber, Hebrew, and Maori and in these languages the inflected auxiliary precedes the verb. ...

Universal [23-a] is false. It is falsified in Basque, Burmese, Burushaski, Finnish, Japanese, Norwegian, Nubian, and Turkish, in which the proper noun precedes the common noun but in which the noun does not precede the genitive.

We found the following previously undiscovered universals in the data.

Universal 1. If in a language the adjective precedes the adverb then the main verb precedes the subordinate verb.

Examples of this universal are 8 languages: Fulani, Guarani, Hebrew, Malay, Swahili, Thai, Yoruba, and Zapotec....

UNIVAUTO thus both states in English its discoveries (new universals+problems) and the supporting evidence that makes these discoveries plausible/valid. Additionally, it provides a general context into which it places these discoveries (in the introductory parts of the generated text), as well as a summary of the findings (in the conclusion part of the generated text).

The readability and self-contained nature of the texts the system normally produces must not be overstated. Some users may prefer to use the output as a "skeleton article" to be subsequently enlarged and edited to fit further stylistic and linguistic needs. ${ }^{4}$

\subsection{Portability}

Some discovery systems model general scientific tasks (for induction, classification, explanation, etc.) and would therefore be readily portable to diverse problems in diverse scientific domains. UNIVAUTO is such a system. It mimics the general task of discovery of (logic) patterns from data, and hence would be applicable not only to language universals discovery, where the objects described in the data are languages, but to any database describing any type of objects, be they linguistic or not. This however applies primarily to its discovery module. The text generation module, as it stands, is less flexible and most probably unportable to a domain outside of universals.

\footnotetext{
${ }^{4}$ Summing up the discussion in the last four subsections, it is interesting to note that, from a design perspective, UNIVAUTO turns out to share mechanisms with systems from other domains, giving further credibility to the analysis proposed by Valdés-Pérez [20]: exhaustive search like MECHEM (operating in chemistry, cf. [19]) or KINSHIP (in linguistics, cf. [10]) and survey of the literature like ARROWSMITH (medicine, cf. [17]) for ensuring novelty; preferring logically stronger claims for ensuring interestingness and testing against qualitatively diverse data for ensuring plausibility like the mathematical system Graffiti ([3]), etc.
} 


\subsection{Insightfulness}

The degree of formalization discovery programs require may result in our deeper understanding of the tasks modeled, esp. if the sciences from which the task is originally taken are not sufficiently formalized. Another source of insightfulness may be the outcomes of discovery programs, in the case when they make conspicuous some overlooked aspects of the results. Both the implementation and use of UNIVAUTO have triggered a number of linguistically important insights. Some of these are worth mentioning here.

First, in the application of the system to linguistic typologies ${ }^{5}$ it was consistently found that a set of non-statistical (in contrast to statistical) universals exists that describes all and only the actually attested types, whereas previous influential authors ([6]), although strong proponents of exceptionless universals, have claimed them insufficient to do the job. This consistency of the system's results could not be chance of course, so that it was only a short step finding the explanation. Indeed, a linguistic typology is equivalent to a propositional function, and therefore, as known from propositional logic, for any propositional function there exists a propositional expression that generates it. As a corollary, for any linguistic typology there exists a set of non-statistical universals, describing all and only its attested types ([15]).

Secondly, our system found alternative sets of (non-statistical) universals describing the same typology. This gave rise to the problem of choosing among alternatives, which was never recognised before. Since linguists have traditionally given preference to simpler descriptions, the problem shaped to "Find simplest solution(s)". This turned out not to be difficult, using a minimal set cover mechanism, that was previously implemented for our KINSHIP program ([10]).

And, thirdly, exploring the 451 language database UPSID with UNIVAUTO has led to the formulation of a phonological principle to the effect that if Phoneme 1 implies Phoneme 2, then both phonemes share at least one feature and, besides, Phoneme 2 never has more features than Phoneme 1. This formulation was made possible only after the system's discovery of all universals of this type valid in the database. The subsequent (machine-aided) representation of the phonemes in terms of their feature structure highlighted this statistically significant pattern, holding in 94.5 per cent of the cases $([16])$.

\section{Conclusion}

UNIVAUTO models an important task in linguistics, synthesizing familiar methods from AI and NLP to make discoveries and verbalize these discoveries. The system performs well and is currently being used in the further study of phonological universals. Previous researchers in machine scientific discovery have not seriously considered extending their systems with text generation components basically because, presumably, their discovery objects are either non-verbally

\footnotetext{
5 A "linguistic typology" states all logically possible types and which of these types are actually attested and which are not.
} 
represented in their respective domains or are not sufficiently numerous to merit verbalization.

Acknowledgment. The writing of this paper was partly supported by contract \#I-813 with the Bulgarian Ministry of Education and Science.

\section{References}

1. Colton, S., Bundy, A., Walsh, T.: On the notion of interestingness in automated mathematical discovery. International Journal of Human-Computer Studies 53(3) (2000) 351-376

2. Croft, W.: Typology and Universals. Cambridge University Press, Cambridge (1990)

3. Fajtlowicz, S.: On conjectures of Graffiti. Discrete mathematics 72 (1988) 113-118

4. Greenberg, J. H.: Some universals of grammar with particular reference to the order of meaningful elements. In: Greenberg, J. H. (ed.): Universals of Language. MIT Press, Cambridge, Mass. (1966) 73-113

5. Xuang, X., Fielder, A.: Presenting machine-found proofs. CADE13, Lecture Notes in Computer Science 1104 221-225

6. Hawkins, J.: Word Order Universals. Academic Press, N.Y. (1983)

7. Jakobson, R.: Kindersprache, Aphasie, und allgemeine Lautgesetze. Almqvist \& Wilksell, Uppsala (1941)

8. Maddieson, I.: Patterns of Sounds. Cambridge University Press, Cambridge (1984)

9. Maddieson, I.: Testing the universality of phonological generalizations with a phonetically specified segment database: results and limitations. Phonetica 48 (1991) 193-206

10. Pericliev, V., Valdés-Pérez, R.: Automatic componential analysis of kinship semantics with a proposed structural solution to the problem of multiple models. Anthropological Linguistics 40(2) (1998) 272-317

11. Pericliev, V.: Further implicational universals in Greenberg's data (a computergenerated article). Contrastive Linguistics 24 (1999) 40-51

12. Pericliev, V.: The prospects for machine discovery in linguistics. Foundations of Science 4(4) (1999) 463-482

13. Pericliev, V.: More statistical implicational universals in Greenberg's data (another computer-generated article). Contrastive Linguistics 25(2) (2000) 115-125

14. Pericliev, V.: A linguistic discovery system that verbalises its discoveries. COLING, 19th International Conference on Computational Linguistics, August 24 September 1, Taipei, Taiwan (2002) 1258-62

15. Pericliev, V.: Economy in formulating typological generalizations. Linguistic Typology 6(1) (2002) 49-68

16. Pericliev, V.: Machine discovery of phonological universals. (2003) (Under submission)

17. Swanson, N.: An interactive system for finding complementary literature: A stimulus to scientific discovery. Artificial Intelligence 91(2) (1997) 183-203

18. Valdés-Pérez, R., Pericliev, V.: Computer Enumeration of Significant Implicational Universals of Kinship Terminology. Cross-Cultural Research: The Journal of Comparative Social Science 33(2) (1999) 162-174

19. Valdés-Pérez, R.: Conjecturing hidden entities via simplicity and conservation laws: Machine discovery in chemistry. Artificial Intelligence 65(2) (1994) 247-280

20. Valdés-Pérez, R.: Principles of human-computer collaboration for knowledge discovery in science. Artificial Intelligence 107 (1999) 335-346 